\title{
Synergistic locoregional chemoradiotherapy using a composite liposome-in-gel system as an injectable drug depot
}

This article was published in the following Dove Press journal:

International Journal of Nanomedicine

I December 2016

Number of times this article has been viewed

\author{
Shruti GuhaSarkar' \\ Kamal Pathak ${ }^{2}$ \\ Niyati Sudhalkar ${ }^{3}$ \\ Prachi More' \\ Jayant Sastri Goda ${ }^{3}$ \\ Vikram Gota ${ }^{2}$ \\ Rinti Banerjee' \\ 'Department of Biosciences and \\ Bioengineering, Indian Institute \\ of Technology Bombay, Mumbai, \\ 2Department of Clinical Pharmacology, \\ ${ }^{3}$ Department of Radiation Oncology, \\ Advanced Centre for Treatment \\ Research and Education in Cancer, \\ Navi Mumbai, Maharashtra, India
}

\begin{abstract}
The use of radiosensitizers in clinical radiotherapy is limited by systemic toxicity. The biopolymeric, biodegradable, injectable liposome-in-gel-paclitaxel (LG-PTX) system was developed for regional delivery of the radiosensitizer paclitaxel (PTX), and its efficacy was evaluated with concurrent fractionated radiation. LG-PTX is composed of nano-sized drug-loaded fluidizing liposomes, which are incorporated into a porous biodegradable gellan hydrogel. This allows enhanced drug permeation while maintaining a localization of the drug depot. LG-PTX had an $\mathrm{IC}_{50}$ of $325 \pm 117 \mathrm{nM}$ in B16F10 melanoma cells, and cytotoxicity with concurrent doses of fractionated radiation showed significant increase in apoptotic cells $(75 \%)$ compared to radiation $(39 \%)$ or LG-PTX (43\%) alone. Peri-tumoral injection in tumor-bearing mice showed PTX localization in the tumor 2 hours after administration, with no drug detected in plasma or other organs. LG-PTX administration with doses of focal radiation $(5 \times 3 \mathrm{~Gy})$ significantly reduced tumor volumes compared to control (6.4 times) and radiation alone (1.6 times) and improved animal survival. LG-PTX thus efficiently localizes the drug at the tumor site and synergistically enhances the effect of concurrent radiotherapy. This novel liposome-in-gel system can potentially be used as a platform technology for the delivery of radiosensitizing drugs to enhance the efficacy of chemoradiotherapy.
\end{abstract}

Keywords: radiosensitizer, hydrogel, regional drug delivery, concurrent radiotherapy, lipid nanocarrier

\section{Introduction}

Radiation therapy (RT) plays an essential role in the multimodal approach toward cancer therapy. ${ }^{1}$ Improving efficacy of radiotherapy either by technical developments (3D-conformal radio therapy or intensity modulated radio therapy) $)^{2,3}$ or by concurrent usage of chemotherapy (cisplatin, paclitaxel [PTX], etc) ${ }^{4,5}$ or targeted agents (eg, cetuximab) has been proven in various studies, ${ }^{6}$ but the utility of multimodal approach to cancer therapy is limited by two fundamental facts: 1) certain proportion of cancer cells are radioresistant, leading to tumor recurrence/relapse which finally affects clinical outcome and 2) toxicity of conventional chemotherapeutic and biological agents when used in conjunction with RT. Attempts to improve outcomes of RT have largely focused on 1) radiation dose escalation, ${ }^{2,7} 2$ ) using altered fraction RT schedules, ${ }^{7-9} 3$ ) sensitizing the radioresistant fraction of tumor cells to conventional doses of radiation by using conventional chemotherapeutic drugs, ${ }^{10}$ and 4) biologically targeting cancer cells specifically with concurrent RT. ${ }^{6,11}$ A highly researched strategy of cancer therapy is the use of radiosensitizers in conjunction with radiotherapy, which are therapeutic moieties that can be used prior to, during, or after radiation to augment its effect. ${ }^{12}$ Chemotherapeutic agents, such as PTX, ${ }^{13}$ have been used to arrest tumor
Correspondence: Rinti Banerjee Department of Biosciences and Bioengineering, Indian Institute of Technology Bombay, Powai, Mumbai 400076, Maharashtra, India

Tel +9l 2225767868

Email rinti@iitb.ac.in 
cells in the radiation-sensitive G2 phase of their cell cycle, thereby enhancing the apoptotic effect of subsequent radiation. ${ }^{14}$ However, the use of systemic PTX is associated with major toxicities ${ }^{15}$ and low drug residence at the tumor site. In such a scenario, the strategy of utilizing nanotechnology in the field of oncology presents huge opportunities to overcome these limitations and improve the therapeutic ratio for effective regional delivery of radiosensitizers. ${ }^{16}$ A number of nano-sized carriers ${ }^{17-19}$ and synthetic poly(ethylene glycol) (PEG)-based gels have been studied as drug carriers ${ }^{20-24}$ but have inherent limitations.

This study developed a novel injectable liposome-in-gelpaclitaxel (LG-PTX) system in which the liposomal carrier for PTX allows sustained drug release at the target site, while the use of gellan as an injectable hydrogel ${ }^{25,26}$ to incorporate the drug-loaded liposomes provides a means to increase residence time of the drug at the tumor site. It also evaluated the in vitro and in vivo radiosensitizing efficacy, pharmacokinetics, and bio-distribution of the injectable liposome-in-gel system loaded with PTX in a radioresistant malignant melanoma cell line (B16F10 murine melanoma). ${ }^{12,27}$ This system has potential use as a platform technology for localized drug delivery in cancers currently being treated with radiotherapy, such as cervical, oral, anal canal, and so on, and can provide a minimally invasive route for prolonged and tumor-specific drug delivery at the tumor site.

\section{Materials and methods}

\section{Materials, cells, and animals}

A Milli-Q water system (Millipore, Bedford, MA, USA), supplied with distilled water, provided high purity water $(18.2 \mathrm{M} \Omega \cdot \mathrm{cm})$ for the experiments. Methanol and chloroform (high-performance liquid chromatography [HPLC] grade) with 99.9\% purity were obtained from Spectrochem (Mumbai, India). Sodium chloride crystals (NaCl, GR grade) with $99.5 \%$ purity were obtained from SRL (Mumbai, India). Soya lecithin (soya phosphatidylcholine, [SPC]) was purchased from Himedia (Mumbai, India). Gellan gum (deacetylated, food grade Kelcogel) was obtained from CP Kelco (Atlanta, GA, USA). Rhodamine 6G dye was obtained from Sigma Aldrich (St Louis, MO, USA).

B16F10 murine melanoma cells obtained from the National Centre for Cell Science (NCCS, Pune, India). Cells were cultured and maintained in complete Dulbecco's Modified Eagle's Medium (DMEM; Himedia) supplemented with 10\% fetal bovine serum (FBS; Himedia), $0.2 \%$ Penicillin-Streptomycin (Pen-strep) or antibiotic, antimycotic solution (Himedia) and cultured at $37^{\circ} \mathrm{C}$ in $5 \% \mathrm{CO}_{2}$ to obtain an adherent monolayer ( $~ 80 \%-90 \%$ confluency) over $72 \mathrm{~h}$ in T25 flasks. Cells were detached using Trypsin-EDTA solution (Himedia).

Female C57BL/6 mice of 4-6 weeks weighing 19-23 g were housed at ACTREC (Tata Memorial Hospital, Navi Mumbai, India), and all experiments were carried out in accordance with the standard Guidelines for the Care and Use of Laboratory Animals (Committee for the Purpose of Control and Supervision of Experiments on Animals (CPCSEA), Government of India). Ethical approval was obtained from the ACTREC Institutional Animal Ethics Committee (IAEC) (project no 22/2011). All efforts were made to minimize animal suffering, and the animals were allowed access to food and water ad libitum.

\section{Preparation of liposome-in-gel system (LG-PTX)}

The LG-PTX formulation was composed of PTX-loaded SPC liposomes incorporated into $0.1 \%(\mathrm{w} / \mathrm{v})$ gellan hydrogel. Liposomes were prepared by thin-film hydration, where SPC lipid was dissolved in 2:1 chloroform:methanol mixture and vacuum evaporated to form a homogeneous film, which was hydrated with $0.9 \% \mathrm{w} / \mathrm{v}$ saline. The liposomes were sonicated for $10 \mathrm{~min}$ to reduce size. Rhodamine-6G dye was added to the saline, and PTX was dissolved along with lipids during thin film formation (1:2 [w/w] drug:lipid). Gellan hydrogel $(0.1 \% \mathrm{w} / \mathrm{v})$ was prepared by adding gellan powder to saline at $60^{\circ} \mathrm{C}-70^{\circ} \mathrm{C}$ and stirring to form a clear solution. For the liposome-in-gel, gellan solution was cooled to $\sim 40^{\circ} \mathrm{C}$ and the liposome suspension (with or without loaded dye or PTX) was stirred into it and cooled.

\section{Characterization}

Size and modal size distribution of liposomes were determined by dynamic light scattering (DLS, BI200SM; Brookhaven, NY, USA), and surface charge was measured from zeta potential with Phase Analysis Light Scattering (ZetaPALS; Brookhaven), respectively. For DLS and ZETA measurements, liposomal suspensions were prepared with a lipid concentration of $2 \mathrm{mg} / \mathrm{mL}$. Modal size distribution of the particles was obtained with NNLS analysis (Brookhaven), while average surface charge was calculated from ten ZETA cycles.

PTX release was carried out in a sink with phosphatebuffered saline (PBS)-trypsin (1\% v/v) and methanol (3:1 $\mathrm{v} / \mathrm{v}$ ), and amount of PTX was estimated using HPLC (Jasco, Tokyo, Japan, characteristic peak at $228 \mathrm{~nm}$ ). Imaging was done using cryo-Field Emission Gun-Transmission electron microscopy (cryo-FEG-TEM; JEM-2100F, JEOL, Peabody, MA, USA) by negative staining of liposomes with 
phosphotungstic acid. Liposome-in-gel samples were flash frozen in liquid nitrogen and lyophilized overnight for scanning electron microscopy (SEM; S-3400N, Hitachi, Tokyo, Japan) of internal morphology. Viscosity of the hydrogel was studied using a viscometer (Physica MCR 301; Anton Paar, Graz, Austria). Degradation studies were carried out by incubation of LG-PTX in PBS-trypsin sink at $37^{\circ} \mathrm{C}$ and measuring weight of remaining gel.

\section{Cellular internalization and cytotoxicity}

Uptake in B16F10 cells was evaluated from incubation with rhodamine-6G dye-loaded liposomes and imaging with confocal laser scanning microscopy (CLSM; Olympus Fluoview FV500, Olympus, Tokyo, Japan). Cells were grown on glass coverslips and incubated with the formulation and free dye for $3 \mathrm{~h}$, after which the excess dye was washed away with PBS; then, the cells were fixed on the glass slides with $10 \%$ formalin, and imaged with CLSM. Average fluorescence intensities were calculated using ImageJ software. Cytotoxicity assay was done with liposome-PTX and LG-PTX on B16F10 cells for $72 \mathrm{~h}$. Cell viability was measured (using sulphorhodamine-B assay) at increasing drug concentrations (10-10,000 nM), and $\mathrm{IC}_{50}$ was calculated using MS Excel and GraphPad.

\section{Cellular cytotoxicity with radiation}

Cells were grouped into Control, Radiation alone, LG-PTX alone, and LG-PTX + Radiation groups. Control cells were fixed at $80 \%$ confluency in $70 \%$ ethanol. For cells with radiation, fractionated radiation was given in steps of 3 Gy daily for 5 days (3-15 Gy) using cobalt-60 gamma rays (Bhabatron-II machine ${ }^{\mathrm{TM}}$ ) at a distance of $80 \mathrm{~cm}$ and $71 \mathrm{cGy} / \mathrm{min}$ dose rate. For cells with LG-PTX alone, LG-PTX (10 $\mu \mathrm{M} /$ day for 5 days) was added without radiation. For the LG-PTX + Radiation group, cells were pretreated with LG-PTX $(10 \mu \mathrm{M}) 2$ hours prior to radiation (3 Gy daily for 5 days). Cells were fixed in $70 \%$ ethanol, re-suspended in PBS along with RNaseA $(10 \mu \mathrm{g} / \mathrm{mL})$, and stained with propidium iodide (PI, $50 \mu \mathrm{g} / \mathrm{mL}$ ) for cell cycle analysis using fluorescence-activated cell sorting (FACS; FACSCalibur ${ }^{\mathrm{TM}}$, BD Biosciences, Franklin Lakes, NJ, USA). FACS results were analyzed using ModFit LT 4.0 to determine percentage apoptosis of cells. The synergy between the effects of radiation and LG-PTX was evaluated by Chou analysis using CompuSyn software (Chou and Martin; ${ }^{; 8,29}$ ).

\section{In vivo biodistribution and efficacy}

Upper thigh of mice was shaved before subcutaneous injection of tumor cells $\left(2 \times 10^{6}\right.$ cells $)$, and tumors were grown to $\sim 100 \mathrm{~mm}^{3}$. LG-PTX was subcutaneously injected using a 0.24 gauge needle into the peri-tumoral region around the subcutaneous murine melanoma tumor developed in the flank of the mice. For drug biodistribution, blood was collected at $0.5 \mathrm{~h}$ and at sacrifice ( $2 \mathrm{~h}$ ). Tumor, skin over the tumor, liver, kidney, and small intestine were analyzed for PTX using HPLC.

\section{Processing of blood and tissue}

Blood sample was collected in K2-EDTA containing vacutainers just before sacrifice by retro-orbital puncture. The samples were centrifuged at 4,000 rpm for $20 \mathrm{~min}$ and plasma thus separated was stored at $-20^{\circ} \mathrm{C}$ for pending analysis. Tumor and skin over the tumor, liver, kidney, and intestine were dissected out and rinsed with PBS and dried on blotting paper. These tissues were then homogenized with 1:4 w/v of PBS. Tissue homogenates were stored at $-80^{\circ} \mathrm{C}$ for pending analysis.

\section{Sample preparation for HPLC analysis}

An amount of 1,000 $\mu \mathrm{L}$ of ice cold acetonitrile was added to $100 \mu \mathrm{L}$ of plasma or tissue homogenate and vortexed for $5 \mathrm{~min}$ followed by centrifugation at $12,000 \mathrm{rpm}$ at $10^{\circ} \mathrm{C}$ for $10 \mathrm{~min}$. Next, $900 \mu \mathrm{L}$ of the supernatant was transferred into new labeled storage vials and dried under nitrogen gas at $42^{\circ} \mathrm{C}$ for $30 \mathrm{~min}$. The sample was then reconstituted with $100 \mu \mathrm{L}$ of reconstitution solution (acetonitrile:water 80:20 v/v) and $30 \mu \mathrm{L}$ was injected into the Dionex UHPLC Ultimate 3000 system fitted with a Kinetex $5 \mu \mathrm{XB}$ C18, 100A, $4.6 \mathrm{~mm}$ (Phenomenex) column maintained at $40^{\circ} \mathrm{C}$. The mobile phase was comprised of acetonitrile and triethylamine (53:47) at an isocratic flow rate of $1 \mathrm{~mL} / \mathrm{min}$. The retention time of PTX was found to be $11.4 \pm 0.5 \mathrm{~min}$. Chromatograms were acquired using the Ultimate 3000RS Diode array detector TM set at $229 \mathrm{~nm}$. Percentage recovery of PTX was $\sim 80 \%$ and found to be similar in plasma and tissues.

For radiosensitization efficacy, when tumor size reached $100 \mathrm{~mm}^{3}$, mice were randomized into three groups (animals per group: untreated control $[\mathrm{n}=12]$, radiation only $[\mathrm{n}=8]$, and LG-PTX with radiation [n=8]). In the LG-PTX with radiation group, LG-PTX was injected peri-tumorally $2 \mathrm{~h}$ before the application of radiation. Fractionated doses of focal radiation $(5 \times 3 \mathrm{~Gy})$ were delivered (under anesthesia) to the tumor every $72 \mathrm{~h}$ by a Cobalt-60 Gamma rays at a source to skin distance (SSD) of $80 \mathrm{~cm}$ and a dose rate of $71 \mathrm{cGy} / \mathrm{min}$. Tumor size was recorded every third day and mice were sacrificed 4 days after the fifth dose (day 19). Control mice were observed up to 19 days, and mortality was documented. Survival was compared between the groups by using Kaplan-Meier method, and $P$-values were computed 
by log rank test. The animals that were alive at the end of observation period in either of the groups were censored. Tumor volumes were calculated before each dose and at sacrifice or death of the animal, and the fold increase in the tumor volume was calculated for each animal compared to its volume before start of the experiment.

Tumor growth inhibition (TGI) was calculated as: $\left(1-\mathrm{RTV}_{\mathrm{T}} / \mathrm{RTV}_{\mathrm{C}}\right) * 100$, where RTV is the relative tumor volume (calculated for each animal as the ratio of tumor volume after the fifth dose $\left[\mathrm{TV}_{5}\right]$ and initial tumor volume $\left[\mathrm{TV}_{0}\right]$, ie, $\mathrm{TV}_{5} / \mathrm{TV}_{0}$ ), $\mathrm{RTV}_{\mathrm{T}}$ is the average RTV for the treatment group, and $\mathrm{RTV}_{\mathrm{C}}$ is the average RTV for the control group.

Cells from the tumors were also separated by incubation with trypsin and then centrifuged to separate tumor cells from the tissue. These cells were then fixed in $70 \%$ ethanol and stained with PI, and cell cycle analysis was done using FACS.

\section{Statistical analysis}

All studies were done in triplicate, and data were presented as mean \pm standard deviation (SD). Statistical significance was calculated using GraphPad software and Student's $t$-test was used, where $P<0.05$ was considered significant.

\section{Results and discussion}

The rationale for radiosensitization with concurrent use of chemotherapy agents is governed by three clinical factors. ${ }^{10}$ First, concomitant chemoradiotherapy can be used with the intent of organ-preservation, resulting in improved cosmesis and organ function. Second, chemotherapy can act as a radiosensitizer, improving the probability of locoregional control (in-field cooperation) and, in some cases, survival, by aiding the destruction of radioresistant clones. Third, chemotherapy given as part of concurrent chemoradiation may act systemically and potentially eradicate distant micro metastases by virtue of spatial cooperation. Conventionally, chemotherapeutic drugs used as radiosensitizers are given systemically (either orally or by intravenous injections). ${ }^{12}$ Systemic administration of chemotherapeutic moieties is associated with numerous toxic side effects and does not facilitate an increase in local drug concentrations or any appreciable retention of the drug at the tumor site, thus necessitating multiple dosage regimens. This problem can be addressed by using regional drug delivery systems that can be applied and retained at the tumor site and can potentially improve cancer treatment outcomes by using adjuvant or postoperative therapies. PTX has been known to be a radiosensitizer for a number of cancers, ${ }^{13}$ since it stabilizes cellular microtubules and can arrest tumor cells in the radio-responsive $\mathrm{G} 2 / \mathrm{M}$ phase of their cell cycle to enhance their response to radiation. However, the commercially available form of the drug has severe side effects and dose-limiting toxicities due to the Cremophor excipient used. ${ }^{15}$ Liposomal formulations of PTX (without concomitant radiation) have previously been studied for intravenous use. ${ }^{30-32}$

\section{Characterization of LG-PTX}

The developed LG-PTX formulation was composed of PTXloaded SPC liposomes incorporated into an injectable gellan hydrogel. The SPC liposomes were found to have sizes of $124 \pm 7 \mathrm{~nm}$ with a unimodal distribution from DLS measurements (Figure 1A), polydispersity of $0.22 \pm 0.01$, and an average negative surface charge of $-16.8 \pm 1.7 \mathrm{mV}$, showing good fit of the experimental phase change data (Figure 1B). SEM imaging of the internal morphology of LG-PTX showed the porous gel structure of the gellan hydrogel $(0.1 \% \mathrm{w} / \mathrm{v})$ (Figure 1C) with pore sizes of $\sim 40-50 \mu \mathrm{m}$, interconnected gellan strands, and the presence of spherical SPC liposomes inside the gel matrix (Figure 1D). High magnification cryoFEG-TEM of the PTX-loaded SPC liposomes showed spherical liposomes of sizes $\sim 200 \mathrm{~nm}$ (Figure 1E) with aqueous cores.

The gellan hydrogel has to have sufficiently low viscosity to allow easy peri-tumoral injection through small gauge needles. Viscosity of LG-PTX (Figure $1 F$ ) was $3.2 \pm 0.5 \mathrm{~Pa} \cdot \mathrm{s}$ (at $0.129 \mathrm{~s}^{-1}$ shear rate) and showed sharp decrease with increasing shear rate, indicating a shear thinning property and that LG-PTX will flow when subjected to shear forces during injection.

PTX is used as a model hydrophobic drug and showed high encapsulation efficiency of $91.2 \% \pm 0.7 \%$ in SPC liposomes, and appreciable drug release at $37^{\circ} \mathrm{C}$ in PBStrypsin/methanol (3:1) medium, with a cumulative release of $72.0 \% \pm 11.6 \%$ over a time span of $72 \mathrm{~h}$ (Figure $1 \mathrm{G}$ ). Since PTX has very low solubility in water, methanol was added to the sink medium in order to aid in its solubilization. The in vitro degradation of LG-PTX shows $63.9 \% \pm 5.1 \%$ loss of weight in $72 \mathrm{~h}$ of incubation (Figure 1H), and images show dispersion and degradation of the formulation over this time (Figure 1I).

SPC is a naturally derived, biocompatible, Generally Recognized As Safe (GRAS) entity ${ }^{33}$ composed of a mixture of phosphoglycerides, a majority of which have unsaturated lipid chains. SPC is known to have a fluidizing effect on lipid packing (such as in cellular membranes) and thus enables 

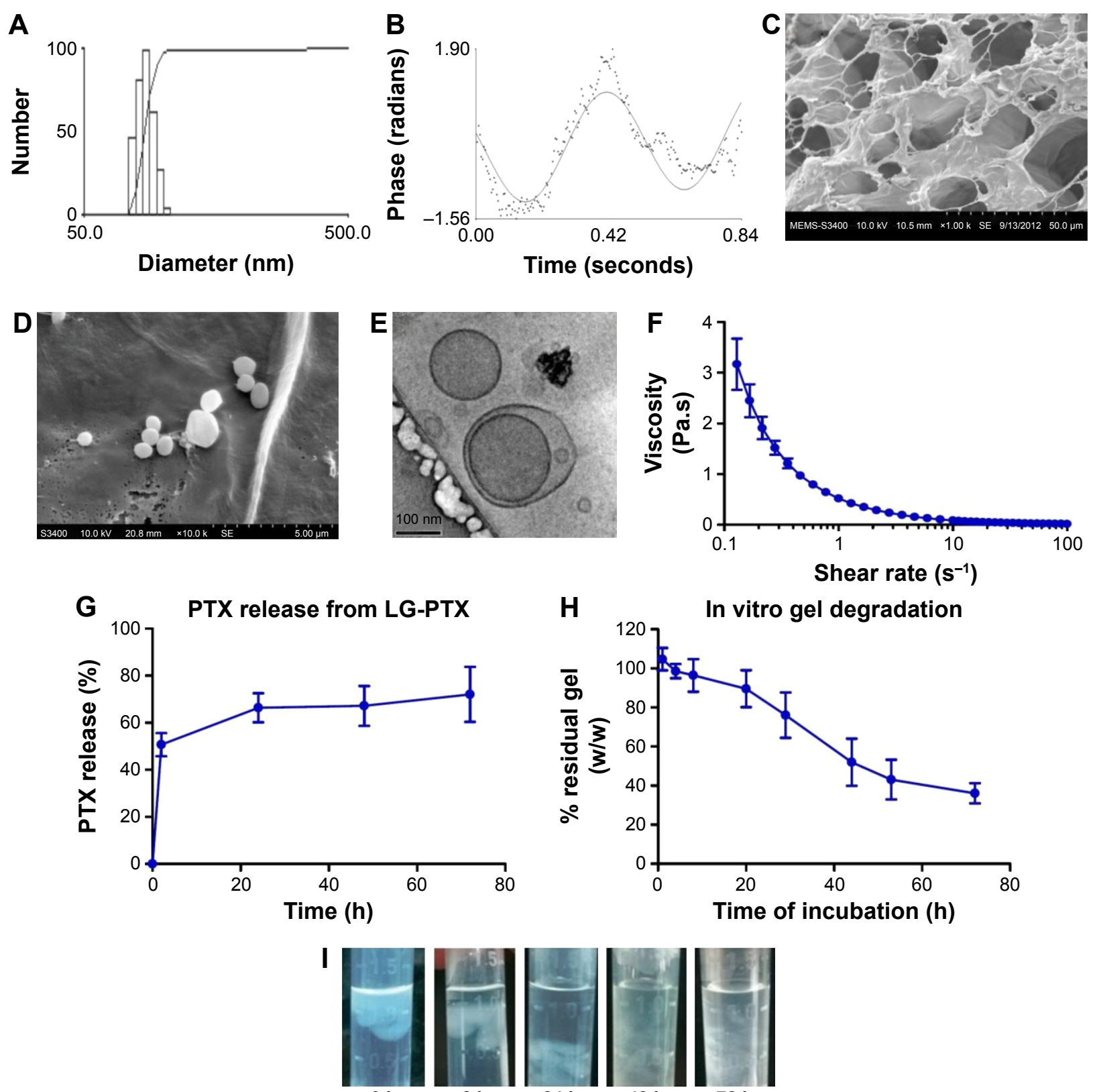

$\mathbf{O h}$
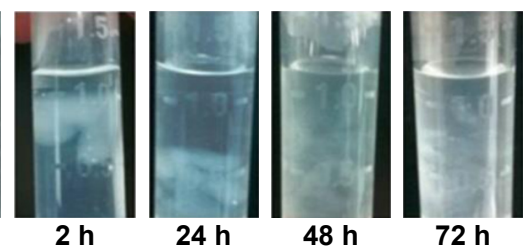

Figure I (A) Size distribution from DLS showing unimodal distribution; (B) ZETA potential measurement of liposomes; (C) SEM image of LG-PTX showing porous gel matrix (scale bar $50 \mu \mathrm{m}$ ) with (D) spherical liposomes inside the gel matrix (scale bar $5 \mu \mathrm{m}$ ); (E) cryo-FEG-TEM images of PTX-loaded SPC liposomes (scale bar I00 nm); (F) viscosity v/s shear rate profile of LG-PTX, showing shear thinning property; (G) cumulative paclitaxel release from LG-PTX over 72 h; (H) degradation profile of LG-PTX over $72 \mathrm{~h}$, and (I) visual observation of LG-PTX during degradation, showing gradual breakdown of the gel.

Abbreviations: DLS, dynamic light scattering; FEG, field-emission gun; SEM, scanning electron microscopy; LG-PTX, liposome-in-gel-paclitaxel; PTX, paclitaxel; SPC, soya phosphatidylcholine; TEM, transmission electron microscope; h, hours.

better uptake into cells. The use of liposomes as a carrier for the radiosensitizing drugs provides a distinct advantage as a platform for efficient entrapment of other hydrophobic radiomodifiers, such as docetaxel, ${ }^{34}$ other taxanes, camptothecin, ${ }^{35}$ prostaglandins, and so on, or a combination of these drugs, can be encapsulated in the lipid bilayers with a high degree of efficiency, as well as have sustained release over time. The injectable bio-polymeric gellan hydrogel $(0.1 \% \mathrm{w} / \mathrm{v})$ used here undergoes gelling via cross-linking with cations as well as by cooling to temperatures $\sim 37^{\circ} \mathrm{C}$, and it is biodegradable and known to be biocompatible. ${ }^{36}$ LG-PTX has large pore sizes that can allow the encapsulated spherical liposomes to diffuse out and be taken up by tumor cells. The use of a hydrogel in the peri-tumoral injection of the LG-PTX formulation provides a biodegradable, porous matrix that can localize the drug-loaded liposomes in the vicinity of the tumor. The gellan hydrogel itself can be used to carry hydrophilic radiosensitizing drugs such as gemcitabine, ${ }^{37}$ 5-flurouracil (5-FU), 
cisplatin, carboplatin, doxorubicin, ${ }^{35}$ and so on, and can potentially function as a dual drug delivery system.

\section{Cellular internalization and cytotoxicity}

As proof of the radiosensitizing property of LG-PTX, the murine melanoma cell line (B16F10) ${ }^{38}$ was used, which is known to be resistant to low, fractionated doses of radiation. ${ }^{12}$ B16F10 cells incubated with rhodamine-6G-loaded liposomes showed bright fluorescence, indicating a high degree of internalization (Figure 2C), and cells with internalized liposomes had significantly higher average fluorescence intensities compared to control cells (Figure 2A) and cells incubated with free dye (Figure 2B), as shown in Figure 2D ( $* P<0.05$ compared to control, $* * P<0.05$ compared to free dye). This showed that the use of liposomes greatly increases cellular internalization of a dye that otherwise has very low uptake and may similarly increase uptake of encapsulated therapeutic moieties. Cytotoxicity on B16F10 cells (Figure 2E) showed that the $\mathrm{IC}_{50}$ for the liposome-PTX was $541 \pm 106 \mathrm{nM}$ and for LG-PTX was $325 \pm 117 \mathrm{nM}$, with no significant difference between $\mathrm{IC}_{50}$ for the liposomes and LG-PTX, showing appreciable cytotoxic activity.

\section{Cellular cytotoxicity with radiation}

The radiosensitizing property of LG-PTX on B16F10 cells with five concurrent doses of 3 Gy radiation every $24 \mathrm{~h}$ was compared to five doses of radiation alone and LG-PTX alone. Figure 3A shows the percentage apoptosis measured normalized to control cells ( $1.9 \% \pm 0.9 \%$ apoptosis, Figure $3 \mathrm{~B})$, and the addition of LG-PTX significantly increases the apoptosis in the cells over the course of five doses of radiation. The LG-PTX-treated group shows significantly higher percentage of apoptotic cells (75\%) from the fourth dose onwards, compared to both the radiation alone (39\%) and the LG-PTX formulation alone $(43 \%)$ treated cells. The results are statistically significant $(P<0.05)$ using two-way analysis of variance.

There is no significant difference in the percent of apoptotic cells obtained with radiation alone at 6 Gy and 9 Gy. However, the increased apoptosis observed with LG-PTX alone at the third dose ( $9 \mathrm{~Gy})$ is due to the cumulative effect of PTX release from the LG-PTX during the use of multiple doses. This trend is continued in the fourth dose (12 Gy) due to the same steady release of PTX from LG-PTX. B16F10 melanoma cell line is also known to be highly radiationresistant, and this may account for apoptosis due to radiation alone being lower than that with LG-PTX alone. Maximal cellular apoptosis is obtained only with the tandem use of LG-PTX and fractionated radiation.

A qualitative comparison of graphs for the three groups (Figure 3C) clearly shows that there is an increase in the percentage of apoptosis (blue peak) with increase in doses of both radiation and LG-PTX and a concurrent decrease in the percentage of cells in G1 phase (red peaks), with a marked effect observed with lower dose in the cells treated with LG-PTX and radiation compared to control, radiation only,
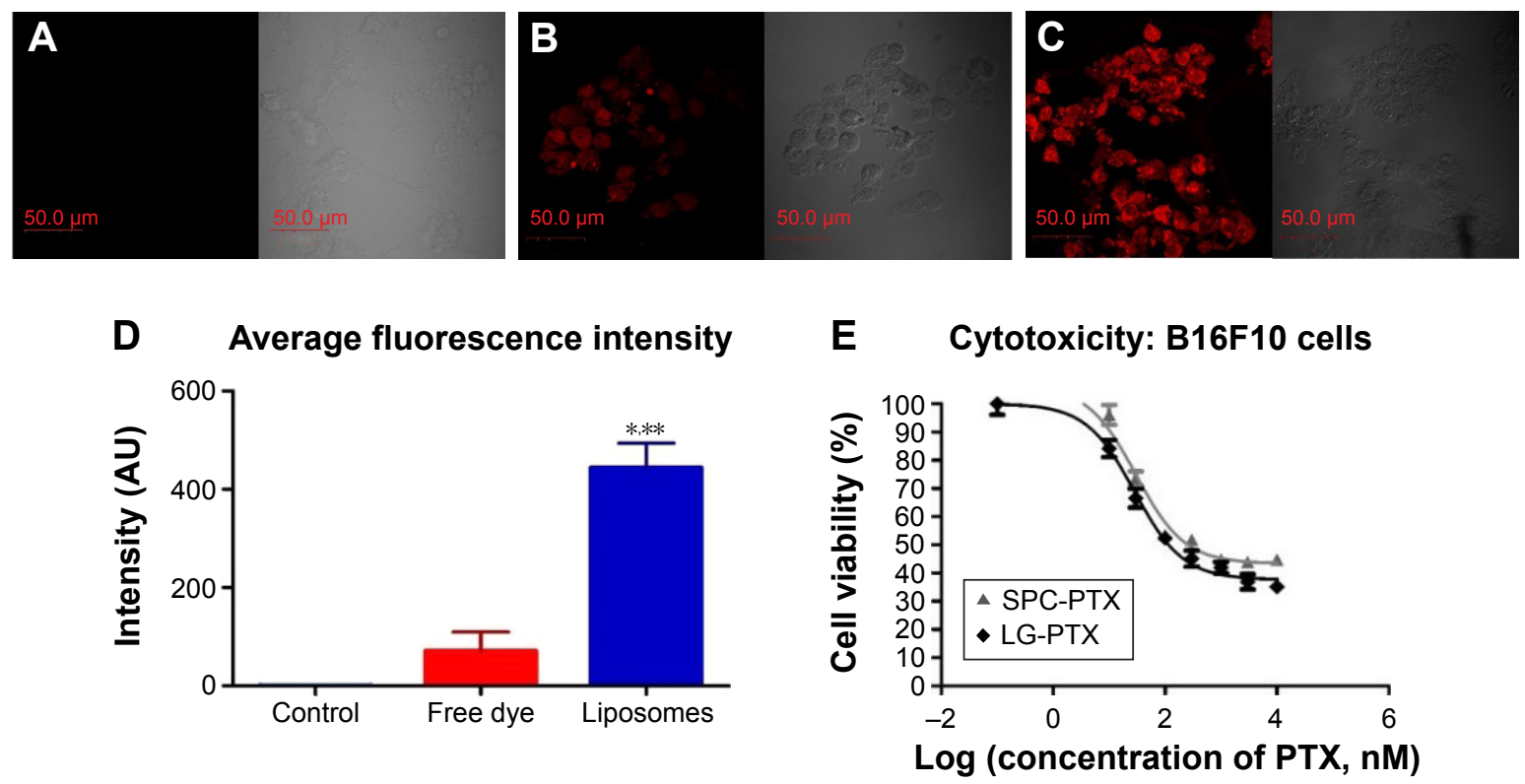

Figure 2 CLSM images (fluorescence and bright field) of (A) control BI6FI0 cells, (B) cells with free dye, and (C) cells with dye-loaded SPC liposomes (scale bar for all images: $50 \mu \mathrm{m}$, magnification 60x); (D) comparison of average fluorescence intensity of cells internalizing free dye and dye loaded in liposomes ( $* P<0.05$ compared to control, **P $<0.05$ compared to free dye); (E) cytotoxicity of formulations on BI6FI0 cells: IC ${ }_{50}$ calculation with SPC-PTX liposomes and LG-PTX formulation.

Abbreviations: CLSM, confocal laser scanning microscopy; LG-PTX, liposome-in-gel-paclitaxel; PTX, paclitaxel; SPC, soya phosphatidylcholine. 


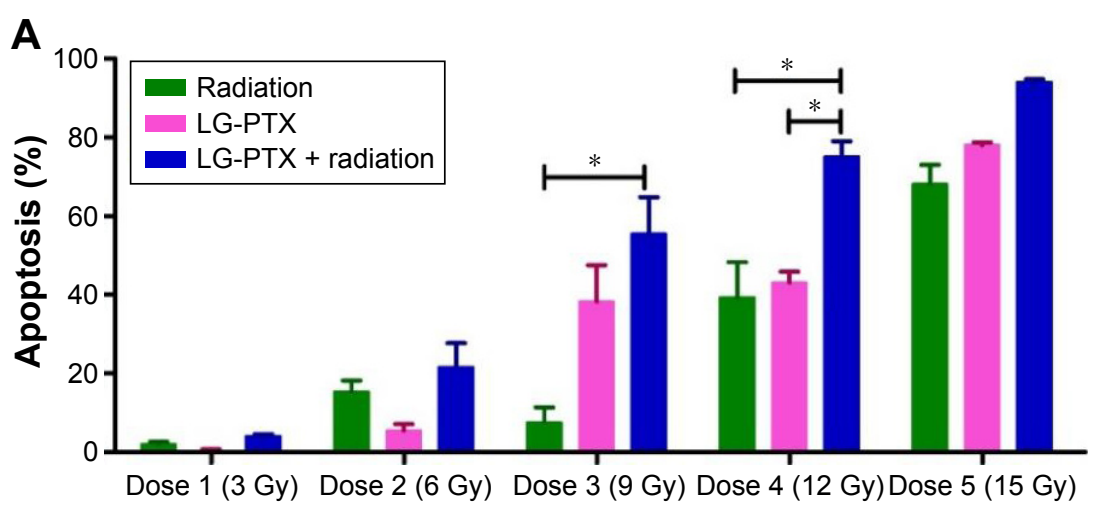

B

Radiation dose

C
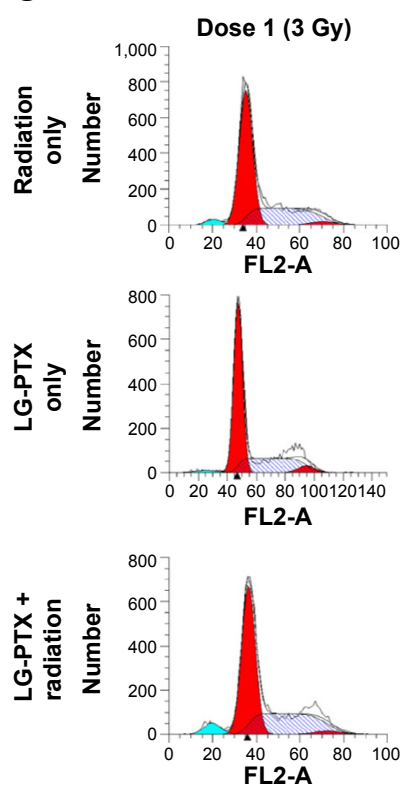

D
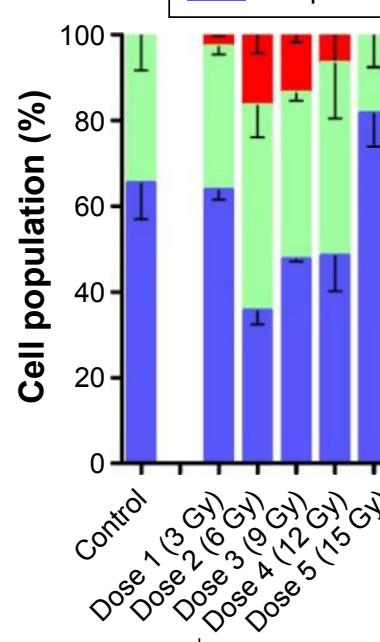

Radiation only
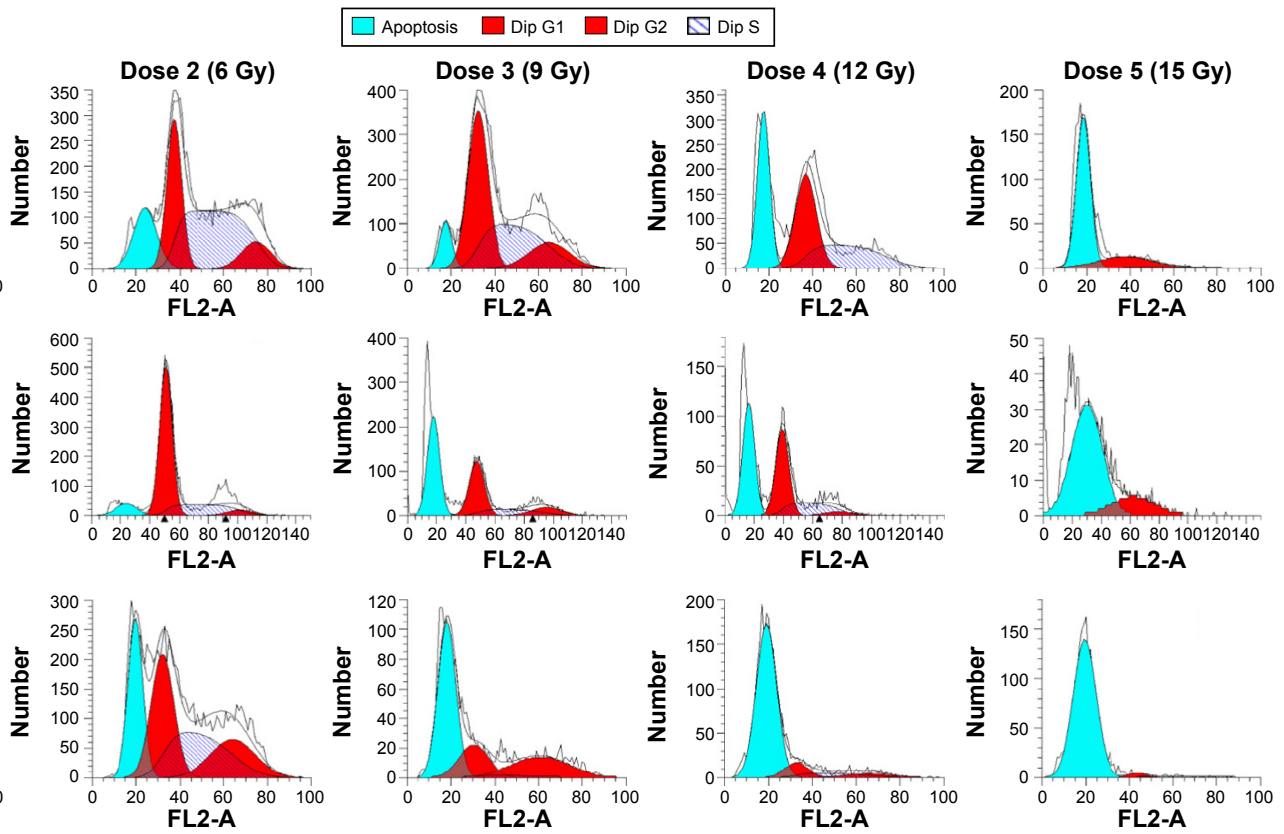

E

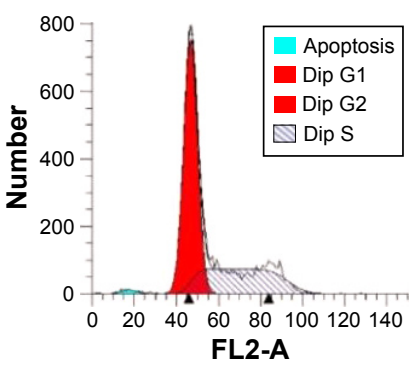

FL2-A 
and LG-PTX only groups. The radiosensitizing property of PTX involves arresting cells in the more radio-responsive G2 phase. This phase is also the most radiosensitive phase of tumor cells, since ionizing radiation is more likely to cause double-stranded DNA breaks. Thus, pre-treatment of the cells with PTX enables a higher percentage of cells to be arrested in the G2/M phase, and subsequent application of radiation increases apoptosis in the cells. PTX thus functions as a radiosensitizer to amplify the apoptotic effect of a dose of radiation.

In the first three doses of LG-PTX and radiation, the percentage of cells in G2 phase is much higher in the LG-PTX + radiation group (33\%) compared to radiation only (14\%) and LG-PTX only (19\%) groups (Figure 3D and E). This shows that the use of LG-PTX increases the number of cells in the G2 phase, and this makes them more susceptible to the subsequent effect of ionizing radiation. From the fourth dose onward, the total number of cells is diminished in all the treatment groups and is composed mostly of radio-resistant cells in the S and early G1 phases (Figure 3D).

In order to determine whether using multiple fractions of radiation instead of one single high dose of radiation had any effect, cells were treated with one single dose of 9 Gy radiation, with and without LG-PTX and compared to cells treated with three fractionated doses of 3 Gy radiation (Figure 4A). The use of fractionated doses with LG-PTX seems to have a very significant $(P<0.05)$ increase in percentage of apoptosis of the cells (57\%), compared to both a single 9 Gy radiation dose with LG-PTX (9\%) and three fractionated doses of 3 Gy radiation alone (7\%), proving the utility of using multiple doses of radiation in tandem with the LG-PTX formulation for therapy. The use of three fractionated doses of 3 Gy radiation along with LG-PTX also serves to increase the percentage of cells arrested in the G2 phase (33\%) (Figure 4B and C), compared to both a single dose of 9 Gy radiation along with LG-PTX (10\%), as well as three doses of 3 Gy radiation alone (13\%), and this helps to enhance the cytotoxic effect of radiation on the cells.

The synergy between the effects of radiation and LG-PTX was evaluated by Chou analysis using CompuSyn software (Chou and Martin ${ }^{28}$ ). The dose-response curves of the radiation only and the combination (LG-PTX and radiation) were plotted (Figure S1), and the combination index (CI) was calculated using a drug:radiation constant combination ratio of $1: 1 .^{29}$ The $\mathrm{CI}$ value at $\mathrm{ED}_{50}$ (effective dose for $50 \%$ effect) was found to be 0.365 , and since CI $<1$ indicates synergy, this shows the synergistic effect of using a combination of radiation and chemotherapy.
This in vitro cytotoxicity assessment with concurrent radiation allows us to optimize drug and radiation dosage required for in vivo experiments. Further optimization of radiation dose is needed using a clonogenic assay to determine dose enhancement factor and explore other aspects of cell death. ${ }^{39,40}$ Smaller doses of radiation are always preferred for in vivo and clinical applications in view of the underlying radiobiological principles (faster recovery of normal cells and tissues, redistribution of tumor cells in the G2/M phase, and reduction in the proportion of hypoxic fraction of tumor cells) and to maximize survival, but such doses either do not produce an appreciable therapeutic effect or increase the frequency and total duration of the radiotherapy regimen, thereby hampering patient compliance. The justification, and considerable advantage, of using fractionated doses of radiation was observed, implying that the use of LG-PTX greatly enhances the therapeutic effect of even low doses of radiation.

\section{In vivo PTX biodistribution}

Amount of PTX in the tumor $2 \mathrm{~h}$ after subcutaneous injection of LG-PTX was calculated to be $343 \pm 283 \mu \mathrm{g} / \mathrm{g}$ of tissue, which is high enough to serve as a radiosensitizer. ${ }^{41}$ PTX was not detected in plasma and other organs of the animal, showing that the formulation effectively localizes the drug at the tumor site only (Figure 5). Previous studies with intravenous injection of Taxol in a subcutaneous murine melanoma model have shown significant PTX distribution in the plasma and other organs (such as liver and lung) at $2 \mathrm{~h},{ }^{17,42}$ while that from LG-PTX showed drug levels only in the tumor. Other references have shown that only a fraction of the intravenous Taxol dose $(<5 \%, \sim 10 \mu \mathrm{g} / \mathrm{g})$ is accumulated in subcutaneous tumors, ${ }^{43,44}$ while a substantial fraction of drug is accumulated in the tumor when LG-PTX is used $(33 \%, \sim 343 \mu \mathrm{g} / \mathrm{g})$. This is a highly advantageous aspect of the liposome-in-gel system, facilitating site-specific drug accumulation, in order to minimize the adverse effects of subsequent radiation on normal tissues.

\section{In vivo efficacy}

The fold increases in tumor volumes over five doses of LG-PTX and radiation (Figure 6A) show that compared to the control animals, both radiation and radiation with LG-PTX groups have significantly lower fold increase in tumor volumes. LG-PTX with radiation group has 6.4 times lower increase in tumor volume compared to control and 1.6 times lower compared to radiation alone (Figure S2A).

Furthermore, the extent of TGI was calculated for the groups, and calculations showed that radiation alone causes 


\section{A Single radiation dose vs fractions}
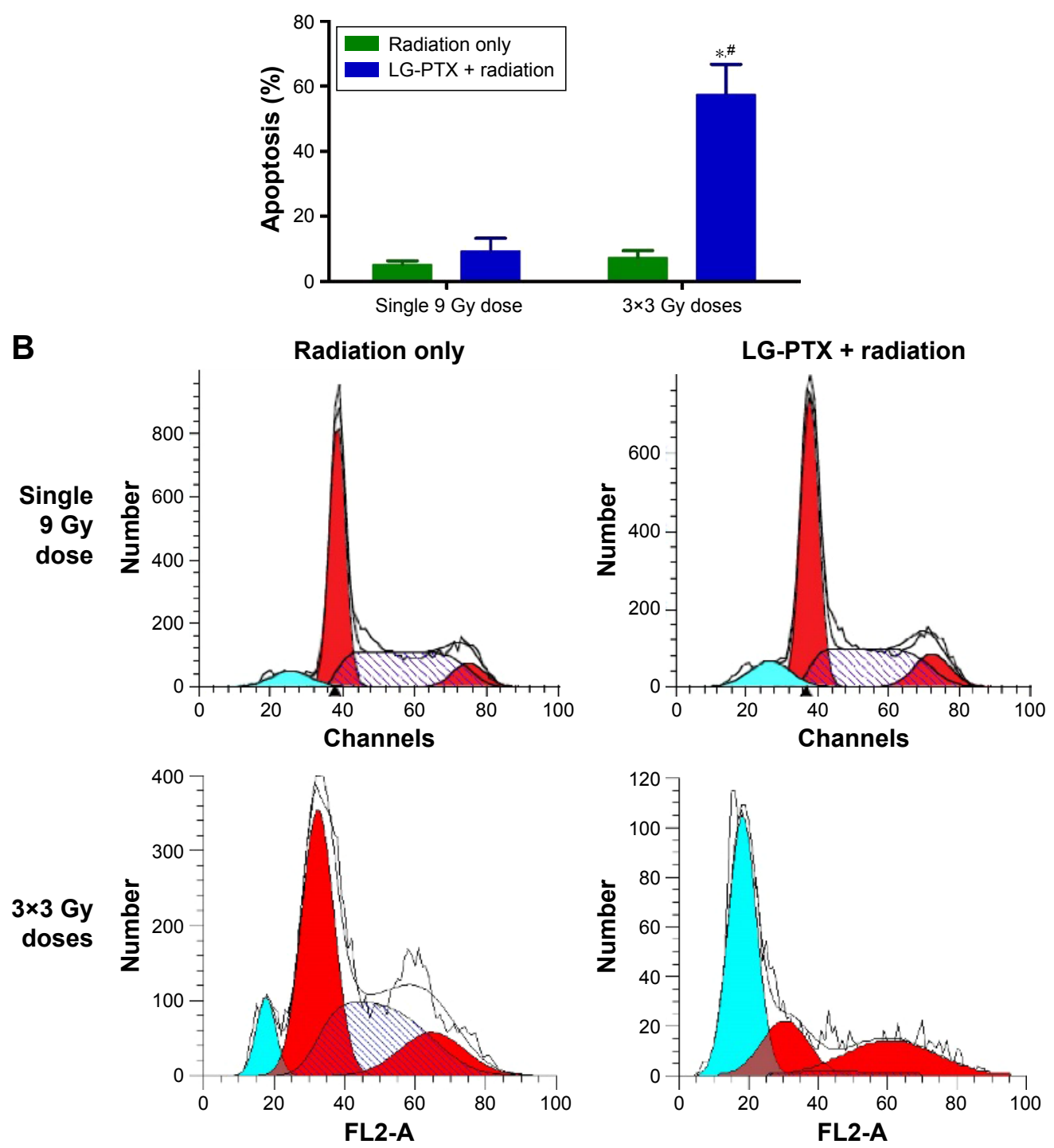

$\square$ Apoptosis $\square \operatorname{Dip}$ G1 $\square \operatorname{Dip}$ G2 ( Dip S

\section{Single vs fractionated RT dose}

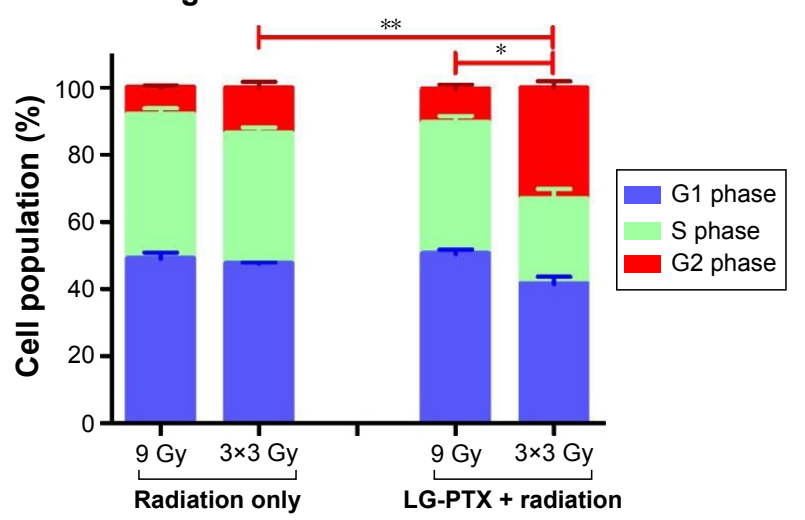

Figure 4 Percentage apoptosis from FACS cell cycle analysis.

Notes: (A) Comparison of single dose versus multiple doses of radiation. $* P<0.05$ in comparison with single 9 Gy LG-PTX + radiation dose; ${ }^{*}<0.05$ in comparison with 3 fractions $(3 \times 3 \mathrm{~Gy}$ ) of radiation only doses. (B) FACS of cells treated with single 9 Gy dose versus $3 \times 3$ Gy doses of radiation and LG-PTX + radiation, showing significantly high apoptosis (blue peak) with LG-PTX and multiple radiation doses ( $3 \times 3 \mathrm{~Gy}$ ) (red peaks indicate cells in GI and G2 phases). (C) Comparison of percentage of cells in GI, S, and G2 phases in cells treated with single and multiple radiation doses. $* P<0.05$ in comparison with single 9 Gy LG-PTX + radiation dose; $* * P<0.05$ in comparison with 3 fractions ( $3 \times 3 \mathrm{~Gy}$ ) of radiation only doses.

Abbreviations: FACS, fluorescence-activated cell sorting; LG-PTX, liposome-in-gel-paclitaxel. 


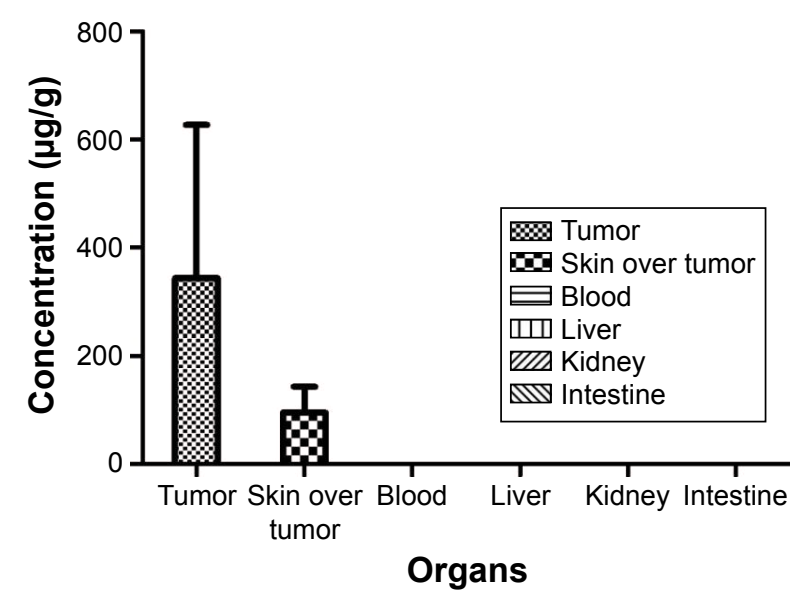

Figure 5 Bio-distribution of paclitaxel in tumor, blood, and other organs 2 hours after peri-tumoral injection of LG-PTX.

Abbreviation: LG-PTX, liposome-in-gel-paclitaxel.

$75.5 \% \pm 4.1 \%$ tumor inhibition, while the formulation and radiation treated group causes a significantly higher inhibition of $84.3 \% \pm 4.2 \%(P<0.05$; Figure $\mathrm{S} 2 \mathrm{~B})$. The survival of the animals (Figure 6B) showed that the survival proportions of the control, radiation, and LG-PTX + radiation groups are $0.5,0.75$, and 1.0 , respectively, indicating improved survival of the animals with the use of the LGPTX formulation. The animal in the radiation only arm died of disease progression and metastasis to distant organs. The dose of PTX used in this study (40 mg/kg) did not cause any adverse toxicity or affect survival of the animals, showing that the LG-PTX formulation is well tolerated in in vivo conditions. From these parameters, it can be inferred that the treatment with the LG-PTX liposome-in-gel formulation causes a significant reduction in tumor volume and an increase in tumor inhibition and greatly improves the survival of the animals.

Cells obtained from the tumors after sacrifice were analyzed with FACS cell cycle analysis, and the percentage of apoptosis observed in the cells from each tumor group (Figure 6C) showed a significantly higher percentage of apoptosis in tumors treated with LG-PTX and radiation, compared to the control and radiation only groups $(* P<0.05$ compared to control, $* * P<0.05$ compared to radiation only). FACS plots (Figure 6D) also show a larger peak for apoptotic cells (blue) and smaller peak for cells in the G1 phase (red), compared to cells from both the control and radiation only groups. This is in agreement with the tumor size data, showing that the use of the LG-PTX formulation in conjunction with radiation has an enhanced effect in tumor inhibition. These results indicate that the LG-PTX + radiation treatment strategy shows a marked improvement in causing apoptosis in the tumor, compared to radiation alone, and confirms the radio-sensitizing effect of the LG-PTX formulation.

Radiation is used as the primary therapeutic measure in a number of cancers, such as glioblastoma, cervical, endometrial, head and neck cancers, and so on, ${ }^{4,45,46}$ which may benefit greatly from localized administration and retention of radiosensitizers at the tumor site. A number of strategies for localized administration of drugs have been developed such as wafers, hydrogels, films, ${ }^{47}$ and microspheres, ${ }^{32}$ using both natural (eg, gelatin and chitosan ${ }^{31,48}$ ) and synthetic polymers (eg, poloxamer and triblock PEG-poly lactic acid-co-glycolic acid PEG-PLGA ${ }^{20,21}$ ) to form injectable drug depots localized at the tumor site, but only Oncoge ${ }^{20}$ and Paclimer $^{32}$ have so far been evaluated in conjunction with radiation. Paclimer (polyphosphoester with PTX) microspheres have shown therapeutic effects in subcutaneous lung cancer xenograft, prostate cancer, ${ }^{47}$ and ovarian cancer via intraperitoneal injection, but were found to cause inflammation and were not evaluated further. ${ }^{32}$ Phase 1 studies with Oncogel, a PTX-loaded thermo-sensitive PLGAPEG-PLGA triblock polymeric gel, in esophageal cancer therapy ${ }^{21}$ has shown favorable outcome, but the versatility of the use of Oncogel is limited by the delicate balance of hydrophilic-hydrophobic groups in the polymer and the attendant stringent methods required for synthesis and drug loading of the formulation. Furthermore, Oncogel acts as a Newtonian fluid and thus requires much higher pressures and longer durations for injection through small bore needles. In comparison, the LG-PTX formulation has a two-step synthesis process and gellan, being a non-Newtonian fluid, shows a reduction in complex viscosity on application of shear forces (Figure 1F), implying that much lower force is needed for clinical injection. The Oncogel formulation can encapsulate only one drug (PTX), but the LG-PTX formulation allows for dual drug loading in the liposomes and the hydrogel matrix.

The novelty of the liposome-in-gel system stems from the use of a bio-polymeric, injectable hydrogel to incorporate drug-loaded liposomes and serves to localize the liposomes at the target site, thereby overcoming the limitations of using liposomes or gels alone. The specific advantages of the LG-PTX system, and its appreciable efficacy in enhancing the therapeutic effect of radiation, suggest that this drug delivery platform can be optimized for use through a multitude of routes, namely, as a locally applied mucosal gel for accessible head and neck cancers and anal verge cancer, intra-vaginal administration for cervical cancers, injected as intra-cranial gels in glioblastomas, intra-esophageal application with an 
A

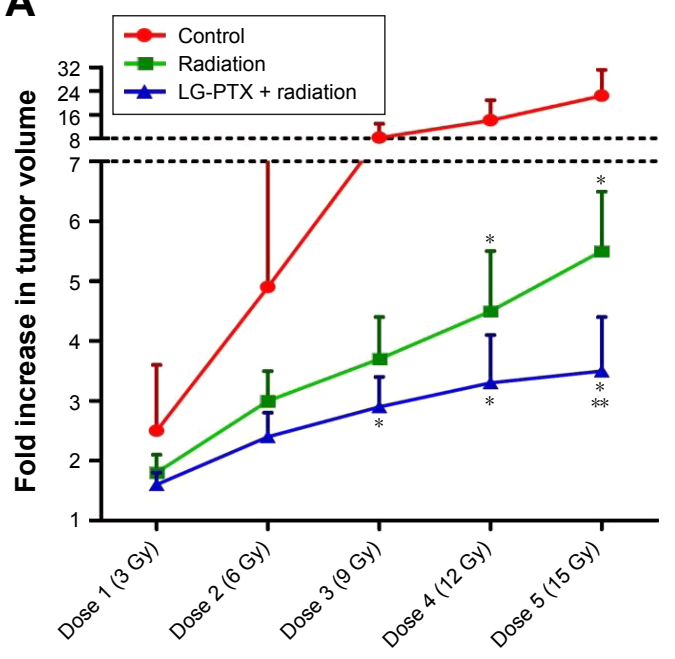

B
Survival proportions

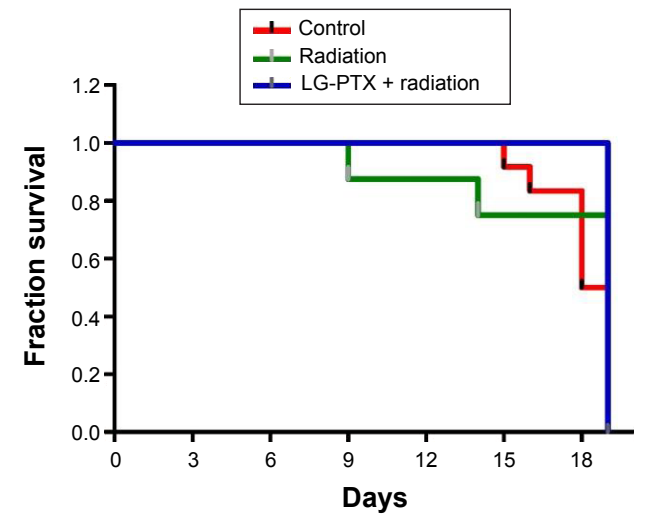

C
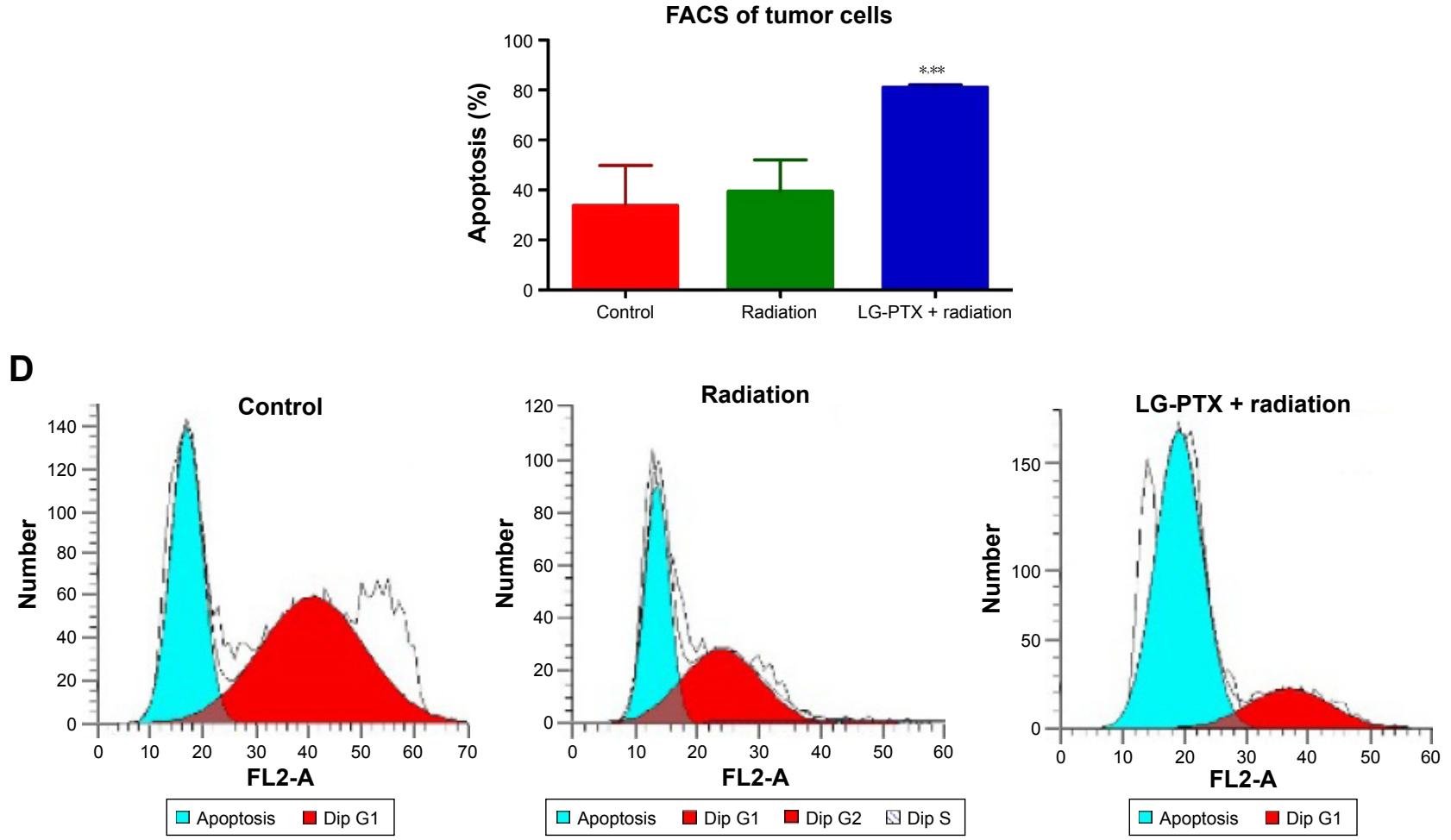

Figure 6 In vivo efficacy.

Notes: (A) Fold increase in tumor volumes in animals in control, radiation, and LG-PTX with radiation groups $(* P<0.05$ compared to control, $* * P<0.05$ compared to radiation). (B) Survival plot of control, radiation, and LG-PTX with radiation animal groups; black/grey vertical bars indicate censored animals. Cell cycle analysis of tumor cells after fifth dose with FACS: (C) percentage apoptosis in the control, radiation, and LG-PTX + radiation animal groups. ( $* P<0.05$ compared to control, $* * P<0.05$ compared to radiation). (D) FACS graph of tumor cells (apoptosis = blue peak; GI phase = red peak).

Abbreviations: FACS, fluorescence-activated cell sorting; LG-PTX, liposome-in-gel-paclitaxel.

endoscope, pancreatic delivery through ultrasound-guided endoscopy, injection into the liver or ovary via laparoscope ${ }^{47}$ and delivery into the lung via bronchoscope. To mimic these clinical uses, a peri-tumoral injection of LG-PTX was chosen, which is less invasive than a direct intratumoral injection. It is of great interest that even when injected peritumorally, very high drug concentrations were observed within the tumor visa-vis the overlying skin and the surrounding normal tissue implying the feasibility of the gel for minimally invasive peritumoral applications in the future. The liposome-in-gel system that has been developed can be further utilized in a versatile manner to incorporate various types of nano-sized 
drug carriers in addition to liposomes, such as cochleates, polymeric nano or micro particles (eg, chitosan and gelatin), and other core-shell particles. These particles can also be functionalized with targeting moieties to enable selective uptake by the targeted tumor cells.

\section{Conclusion}

The liposome-in-hydrogel formulation thus shows efficacy as a regional drug delivery system for the radiosensitizing drug PTX and provides a novel approach to augmenting radiotherapy through the use of a biodegradable gelling system that can localize therapeutic moieties at the target tumor. Although there have been a few studies evaluating gels for the delivery of radiosensitizers, to the best of our knowledge, the present study is the first to report a liposome-in-gel system for regional sustained delivery of a radiosensitizer to augment the synergistic effects of chemoradiation. The liposome-in-gel system acts as an injectable platform for co-delivery of one or more radiosensitizers, both hydrophobic and hydrophilic in nature. The technology has the potential to increase the effectiveness of radiotherapy with the local controlled release of radiosensitizers without the systemic toxicity associated with intravenous delivery.

\section{Acknowledgment}

This work was funded by a seed grant from the Healthcare Consortium, Indian Institute of Technology Bombay, Mumbai, India.

\section{Disclosure}

The authors report no conflicts of interest in this work.

\section{References}

1. Delaney G, Jacob S, Featherstone C, Barton M. The role of radiotherapy in cancer treatment: estimating optimal utilization from a review of evidence-based clinical guidelines. Cancer. 2005;104(6): 1129-1137.

2. Michalski J, Winter K, Roach M, et al. Clinical outcome of patients treated with $3 \mathrm{D}$ conformal radiation therapy (3D-CRT) for prostate cancer on RTOG 9406. Int J Radiat Oncol. 2012;83(3):e363-e370.

3. Sia J, Joon DL, Viotto A, et al. Toxicity and long-term outcomes of dose-escalated intensity modulated radiation therapy to $74 \mathrm{~Gy}$ for localised prostate cancer in a single Australian centre. Cancers (Basel). 2011;3(3):3419-3431.

4. Shang J, Gu J, Han Q, Xu Y, Yu X, Wang K. Chemoradiotherapy is superior to radiotherapy alone after surgery in advanced squamous cell carcinoma of the head and neck: a systematic review and meta-analysis. Int J Clin Exp Med. 2014;7(9):2478-2487.

5. Petrelli F, Coinu A, Riboldi V, et al. Concomitant platinum-based chemotherapy or cetuximab with radiotherapy for locally advanced head and neck cancer: a systematic review and meta-analysis of published studies. Oral Oncol. 2014;50(11):1041-1048.

6. Bonner JA, Harari PM, Giralt J, et al. Radiotherapy plus cetuximab for squamous-cell carcinoma of the head and neck. N Engl J Med. 2006; 354(6):567-578.
7. Baumann M, Herrmann T, Koch R, et al. Final results of the randomized phase III CHARTWEL-trial (ARO 97-1) comparing hyperfractionatedaccelerated versus conventionally fractionated radiotherapy in non-small cell lung cancer (NSCLC). Radiother Oncol. 2011;100(1): 76-85.

8. Overgaard J, Hansen HS, Specht L, et al. Five compared with six fractions per week of conventional radiotherapy of squamous-cell carcinoma of head and neck: DAHANCA 6 and 7 randomised controlled trial. Lancet. 2003;362(9388):933-940.

9. Haslett K, Pöttgen C, Stuschke M, Faivre-Finn C. Hyperfractionated and accelerated radiotherapy in non-small cell lung cancer. $J$ Thorac Dis. 2014;6(4):328-335.

10. Green J, Kirwan J, Tierney J, et al. Concomitant chemotherapy and radiation therapy for cancer of the uterine cervix. Cochrane Database Syst Rev. 2001;(4):CD002225.

11. Rischin D, Peters L, Fisher R, et al. Tirapazamine, cisplatin, and radiation versus fluorouracil, cisplatin, and radiation in patients with locally advanced head and neck cancer: a randomized phase II trial of the Trans-Tasman Radiation Oncology Group (TROG 98.02). J Clin Oncol. 2005;23(1):79-87.

12. Poggi MM, Coleman CN, Mitchell JB. Sensitizers and protectors of radiation and chemotherapy. Curr Probl Cancer. 2001;25(6):334-411.

13. Blajeski AL, Kottke TJ, Kaufmann SH. A multistep model for paclitaxel-induced apoptosis in human breast cancer cell lines. Exp Cell Res. 2001;270(2):277-288.

14. Milross CG, Mason KA, Hunter NR, et al. Enhanced radioresponse of paclitaxel-sensitive and -resistant turnouts in vivo. Eur J Cancer Part A. 1997;33(8):1299-1308.

15. Marupudi NI, Han JE, Li KW, Renard VM, Tyler BM, Brem H. Paclitaxel: a review of adverse toxicities and novel delivery strategies. Expert Opin Drug Saf. 2007;6(5):609-621.

16. Estanqueiro M, Amaral MH, Conceição J, Sousa Lobo JM. Nanotechnological carriers for cancer chemotherapy: the state of the art. Colloids Surf B Biointerfaces. 2015;126:631-648.

17. Joshi N, Saha R, Shanmugam T, Balakrishnan B, More P, Banerjee R. Carboxymethyl-chitosan-tethered lipid vesicles: hybrid nanoblanket for oral delivery of paclitaxel. Biomacromolecules. 2013;14(7): 2272-2282.

18. Nigam A. Nanoparticle paclitaxel (Nanoxel) as a safe and cost-effective radio-sensitizer in locally advanced head and neck carcinoma. J Cancer Ther. 2012;3(1):44-46.

19. Jung J, Kim MS, Park SJ, et al. Enhancement of radiotherapeutic efficacy by paclitaxel-loaded $\mathrm{pH}$-sensitive block copolymer micelles. J Nanomater. 2012 (2012), Article ID 867036, 5 pages.

20. Elstad NL, Fowers KD. OncoGel (ReGel/paclitaxel) - clinical applications for a novel paclitaxel delivery system. Adv Drug Deliv Rev. 2009; 61(10):785-794.

21. Aaron DuVall G, Tarabar D, Seidel RH, Elstad NL, Fowers KD. Phase 2: a dose-escalation study of OncoGel (ReGel/paclitaxel), a controlledrelease formulation of paclitaxel, as adjunctive local therapy to external-beam radiation in patients with inoperable esophageal cancer. Anticancer Drugs. 2009;20(2):89-95.

22. Lee JY, Kim KS, Kang YM, et al. In vivo efficacy of paclitaxel-loaded injectable in situ-forming gel against subcutaneous tumor growth. Int J Pharm. 2010;392(1-2):51-56.

23. Yasui H, Takeuchi R, Nagane M, et al. Radiosensitization of tumor cells through endoplasmic reticulum stress induced by PEGylated nanogel containing gold nanoparticles. Cancer Lett. 2014;347(1): 151-158.

24. Nsereko S, Amiji M. Localized delivery of paclitaxel in solid tumors from biodegradable chitin microparticle formulations. Biomaterials. 2002;23(13):2723-2731.

25. Da Silva LP, Cerqueira MT, Sousa RA, Reis RL, Correlo VM, Marques AP. Engineering cell-adhesive gellan gum spongy-like hydrogels for regenerative medicine purposes. Acta Biomater. 2014;10(11):4787-4797.

26. Morris ER, Nishinari K, Rinaudo M. Gelation of gellan - a review. Food Hydrocoll. 2012;28(2):373-411. 
27. Overwijk WW, Restifo NP. B16 as a mouse model for human melanoma. Curr Protoc Immunol. 2001; Chapter 20:Unit 20.1.

28. CompuSyn software free for download. Available from: http://www. combosyn.com/. Accessed December 31, 2014.

29. Monk BJ, Burger RA, Parker R, Radany EH, Redpath L, Fruehauf JP. Development of an in vitro chemo-radiation response assay for cervical carcinoma. Gynecol Oncol. 2002;87(2):193-199.

30. Fetterly GJ, Straubinger RM. Pharmacokinetics of paclitaxel-containing liposomes in rats. AAPS PharmSci. 2003;5(4):E32.

31. Dhanikula AB, Singh DR, Panchagnula R. In vivo pharmacokinetic and tissue distribution studies in mice of alternative formulations for local and systemic delivery of paclitaxel: gel, film, prodrug, liposomes and micelles. Curr Drug Deliv. 2005;2(1):35-44.

32. Armstrong DK, Fleming GF, Markman M, Bailey HH. A phase I trial of intraperitoneal sustained-release paclitaxel microspheres (Paclimer?) in recurrent ovarian cancer: A Gynecologic Oncology Group study. Gynecol Oncol. 2006;103(2):391-396.

33. USFDA Database of Select Committee on GRAS Substances (SCOGS) Reviews Report No. 106, ID Code 8002-43-5.

34. Alvarez EA, Wolfson AH, Pearson JM, et al. A phase I study of docetaxel as a radio-sensitizer for locally advanced squamous cell cervical cancer. Gynecol Oncol. 2009;113(2):195-199.

35. Kvols LK. Radiation sensitizers: a selective review of molecules targeting DNA and non-DNA targets. J Nucl Med. 2005;46:187S-190S.

36. USFDA 21CFR172.665: Food Additives Permitted for Direct Addition to Food for Human Consumption: Gellan Gum.

37. Pauwels B, Korst AEC, Lardon F, Vermorken JB. Combined modality therapy of gemcitabine and radiation. Oncologist. 2005;10(1):34-51.

38. Lugade AA, Moran JP, Gerber SA, Rose RC, Frelinger JG, Lord EM. Local radiation therapy of $\mathrm{B} 16$ melanoma tumors increases the generation of tumor antigen-specific effector cells that traffic to the tumor. J Immunol. 2005;174(12):7516-7523.

39. Dunne AL, Price ME, Mothersill C, McKeown SR, Robson T, Hirst DG. Relationship between clonogenic radiosensitivity, radiation-induced apoptosis and DNA damage/repair in human colon cancer cells. $\mathrm{Br} J$ Cancer. 2003;89(12):2277-2283.
40. Plesca D, Mazumder S, Almasan A. DNA damage response and apoptosis. Methods Enzymol. 2008;446:107-122.

41. Zanelli GD, Quaia M, Robieux I, et al. Paclitaxel as a radiosensitiser: a proposed schedule of administration based on in vitro data and pharmacokinetic calculations. Eur J Cancer. 1997;33(3):486-492.

42. Zhang W, Shi Y, Chen Y, Hao J, Sha X, Fang X. The potential of pluronic polymeric micelles encapsulated with paclitaxel for the treatment of melanoma using subcutaneous and pulmonary metastatic mice models. Biomaterials. 2011;32(25):5934-5944.

43. Zheng J, Wan Y, Elhissi A, Zhang Z, Sun X. Targeted paclitaxel delivery to tumors using cleavable PEG-conjugated solid lipid nanoparticles. Pharm Res. 2014;31(8):2220-2233.

44. Chen Y, Zhang W, Huang Y, Gao F, Fang X. In vivo biodistribution and anti-tumor efficacy evaluation of doxorubicin and paclitaxelloaded pluronic micelles decorated with c(RGDyK) peptide. PLoS One. 2016;11(3):e0149952.

45. McMeekin DS, Walker JL, Hartenbach EM, Bookman MA, Koh WJ. Phase I trial of the treatment of high-risk endometrial cancer with concurrent weekly paclitaxel and cisplatin and whole abdominal radiation therapy: a Gynecologic Oncology Group study. Gynecol Oncol. 2009; 112(1):134-141.

46. Candelaria M, Garcia-Arias A, Cetina L, Dueñas-Gonzalez A. Radiosensitizers in cervical cancer. Cisplatin and beyond. Radiat Oncol. 2006; $1: 15$.

47. Wolinsky JB, Colson YL, Grinstaff MW. Local drug delivery strategies for cancer treatment: gels, nanoparticles, polymeric films, rods, and wafers. J Control Release. 2012;159(1):14-26.

48. Okino H, Maeyama R, Manabe T. Trans-tissue, sustained release of gemcitabine from photocured gelatin gel inhibits the growth of heterotopic human pancreatic tumor in nude mice. Clin Cancer Res. 2003;9: 5786-5793. 


\section{Supplementary materials}

This section provides the details regarding additional figures of results.

\section{Results}

\section{Cytotoxicity with radiation}

From Figure 3A, the ED50 (effective dose) was plotted as shown in Figure S1. The figure shows in a comparison of the fraction of viable cells $\left(\mathrm{F}_{\mathrm{a}}\right)$ when cells were subjected to radiation and LG-PTX + radiation. The radiation dose at $\mathrm{F}_{\mathrm{a}}=0.5$ was 14.12 Gy for radiation alone and 7.48 Gy for LG-PTX + radiation, showing an appreciable synergistic effect.

\section{In vivo efficacy}

The tumor growth inhibition (TGI) was calculated from the tumor volumes of the animals in the three groups (control, radiation, and LG-PTX + radiation). Figure S2 shows the tumor volumes after the fifth dose was applied (Figure S2A), and the calculated TGI values for radiation and LG-PTX + radiation groups (Figure S2B).

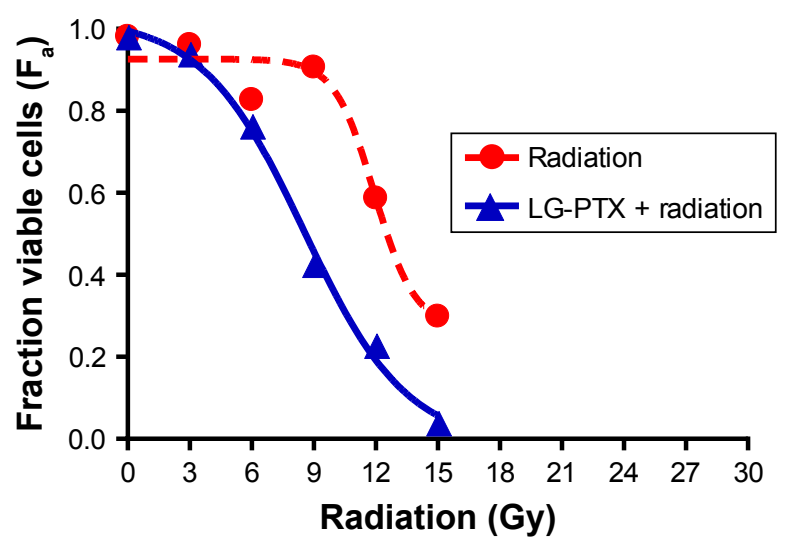

Figure SI Dose-response curve for cytotoxicity with radiation and LG-PTX + radiation. Abbreviation: LG-PTX, liposome-in-gel-paclitaxel.
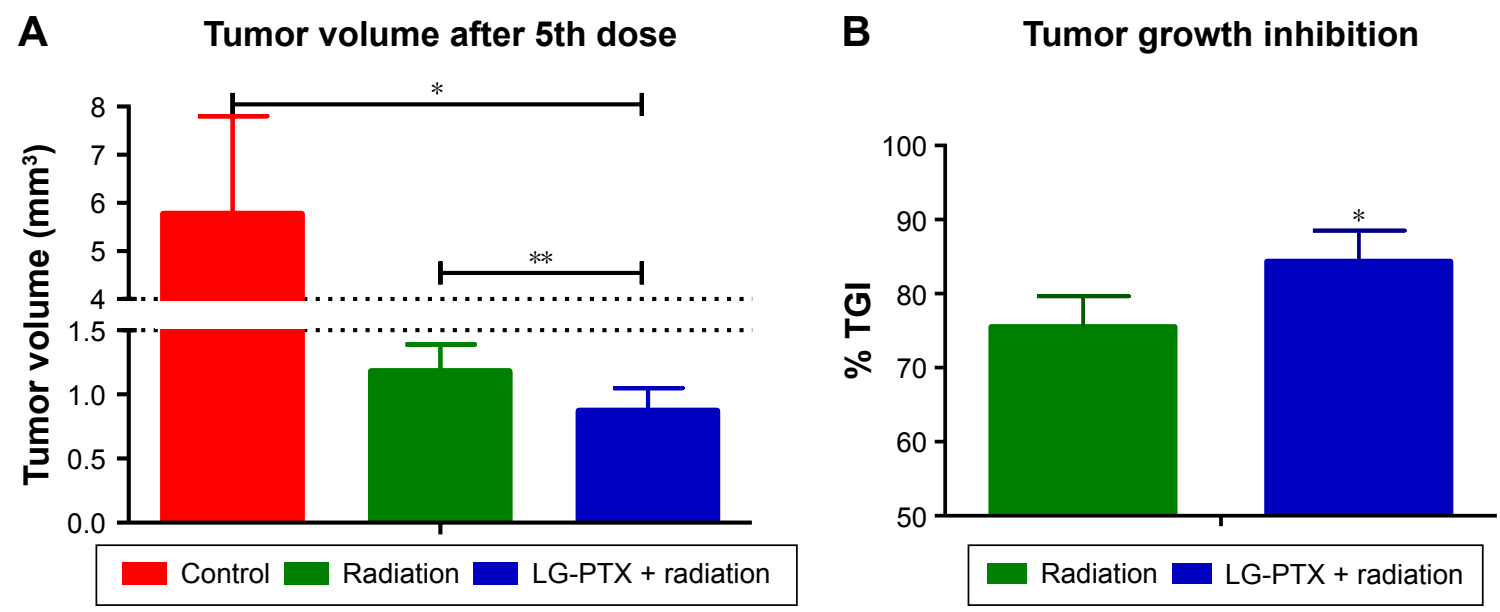

Figure S2 (A) Tumor volume after fifth dose for mice in control, radiation and LG-PTX + radiation groups $(* P<0.05$, $* * P<0.05)$. (B) Tumor growth inhibition (TGI) for radiation and LG-PTX + radiation groups $(* P<0.05)$.

Abbreviation: LG-PTX, liposome-in-gel-paclitaxel.

\section{Publish your work in this journal}

The International Journal of Nanomedicine is an international, peerreviewed journal focusing on the application of nanotechnology in diagnostics, therapeutics, and drug delivery systems throughout the biomedical field. This journal is indexed on PubMed Central, MedLine, CAS, SciSearch $®$, Current Contents ${ }^{\circledR} /$ Clinical Medicine,
Journal Citation Reports/Science Edition, EMBase, Scopus and the Elsevier Bibliographic databases. The manuscript management system is completely online and includes a very quick and fair peer-review system, which is all easy to use. Visit http://www.dovepress.com/ testimonials.php to read real quotes from published authors. 